Astronomical Journal, in press

\title{
The Stellar and Gaseous Contents of the Orion Dwarf Galaxy
}

\author{
John M. Cannon, Korey Haynes, Hans Most \\ Department of Physics \& Astronomy, Macalester College, 1600 Grand Avenue \\ Saint Paul, MN 55105 \\ jcannon@macalester. edu \\ John J. Salzer \\ Astronomy Department, Wesleyan University, Middletown, CT 06457 \\ Department of Astronomy, Indiana University, 727 East Third Street, Bloomington, IN \\ 47405 \\ Kaitlin Haugland \\ Griffith Observatory, 2800 East Observatory Road, Los Angeles, CA 90027 \\ Department of Physics $\&$ Astronomy, Macalester College, 1600 Grand Avenue, Saint Paul, \\ MN 55105 \\ Jillian Scudder \\ Department of Physics $\&$ Astronomy, University of Victoria, PO Box 3055, STN CSC, \\ Victoria, BC, V8W 3P6 Canada \\ Department of Physics \&3 Astronomy, Macalester College, 1600 Grand Avenue, Saint Paul, \\ MN 55105 \\ Arthur Sugden \\ Department of Biology and Medicine, Brown University, Providence, RI 02912 \\ Department of Astronomy, Indiana University, 727 East Third Street, Bloomington, IN \\ 47405 \\ Jacob Weindling \\ Department of Physics \& Astronomy, Macalester College, 1600 Grand Avenue, Saint Paul, \\ MN 55105
}




\begin{abstract}
We present new KPNO 0.9-m optical and VLA H I spectral line observations of the Orion dwarf galaxy. This nearby ( $\mathrm{D} \simeq 5.4 \mathrm{Mpc})$, intermediate-mass $\left(\mathrm{M}_{\mathrm{dyn}} \simeq 1.1 \times 10^{10} \mathrm{M}_{\odot}\right)$ dwarf displays a wealth of structure in its neutral ISM, including three prominent "hole/depression" features in the inner H I disk. We explore the rich gas kinematics, where solid-body rotation dominates and the rotation curve is flat out to the observed edge of the $\mathrm{H}$ I disk $(\sim 6.8 \mathrm{kpc})$. The Orion dwarf contains a substantial fraction of dark matter throughout its disk: comparing the $4.7 \times 10^{8} \mathrm{M}_{\odot}$ of detected neutral gas with estimates of the stellar mass from optical and near-infrared imaging $\left(3.7 \times 10^{8} \mathrm{M}_{\odot}\right)$ implies a mass-tolight ratio $\simeq 13$. New $\mathrm{H} \alpha$ observations show only modest-strength current star formation $\left(\sim 0.04 \mathrm{M}_{\odot} \mathrm{yr}^{-1}\right)$; this star formation rate is consistent with our 1.4 $\mathrm{GHz}$ radio continuum non-detection.
\end{abstract}

Subject headings: galaxies: evolution — galaxies: dwarf — galaxies: irregular — galaxies: individual (Orion dwarf)

\title{
1. Introduction
}

Dwarf galaxies are excellent laboratories for studying the processes that shape galaxy evolution in canonically "simple" conditions. They lack the rotational shear intrinsic to spirals, meaning that star formation (SF) is initiated primarily by local mechanisms. Further, the structures in the ISM are not immediately destroyed, allowing one to study the interplay between SF and the ISM. Targeted observations and larger scale surveys have demonstrated the diagnostic power of $\mathrm{H}$ I spectral line imaging of nearby dwarf systems (e.g., Puche et al. 1992; Puche \& Westpfahl 1994; Hunter et al. 2007; Oh et al. 2008; Walter et al. 2008).

Nearby, gas-rich dwarf galaxies also offer comparatively "simple" environments in which to perform detailed kinematic analysis. Many dwarfs display solid-body rotation that is wellsuited to precision rotation curve work (e.g., Weldrake et al. 2003). Using simple dynamical

arguments, most nearby systems appear to be dark-matter dominated (Mateo 1998), making them important laboratories for understanding the missing baryons problem.

Numerous investigations of nearby systems have revealed rich kinematic structure in the form of shells and holes in the neutral gas (e.g., Kim et al. 1999; Walter \& Brinks 1999, 2001; Ott et al. 2001; to name just a few). It is commonly assumed that these structures result from "feedback" processes (i.e., stellar winds and SNe; e.g., Tenorio-Tagle \& Bodenheimer 
1988). However, debate continues on whether all such features arise from simple stellar evolution processes (e.g., Rhode et al. 1999; Sánchez-Salcedo 2002).

Here, we present new $\mathrm{H}$ I and optical observations of the Orion dwarf galaxy. Originally discovered by Giovanelli (1979), subsequent Arecibo observations showed it to be H I-rich (Springob et al. 2005). However, the low Galactic latitude $\left(-12.3^{\circ}\right)$ and high associated foreground reddening $\left(A_{R}=1.959\right.$ mag; Schlegel et al. 1998) have apparently conspired to keep this nearby system away from detailed observational scrutiny to date. Below we discuss the first spatially resolved study of the ISM and stars within the Orion dwarf; we find modest-level SF in a dark-matter dominated disk that harbors a wealth of small-scale structure.

The distance of the Orion dwarf remains uncertain, primarily due to its low galactic latitude and the resulting difficulty of resolved stellar population work. Previous authors have suggested distances based on the brightest stars method after solving for foreground extinction; for example, Karachentsev \& Musella (1996) find D =6.4 Mpc. More recent infrared work by Vaduvescu et al. (2005) lowers this value to $5.4 \mathrm{Mpc}$; we adopt this latter value in the present work, but note that the distance remains a significant source of uncertainty. We summarize the basic parameters of the Orion dwarf in Table 1 .

\section{Observations and Data Reduction}

\section{1. $\quad K P N O 0.9 m$ Imaging}

New optical images of the Orion dwarf were acquired with the KPNO $0.9 \mathrm{~m}$ telescope1. Two 20-minute exposures in a narrowband $\mathrm{H} \alpha$ filter, and one 4-minute exposure in a broadband R filter, were acquired on 11 March, 2008. Figure1 presents the R-band and continuumsubtracted $\mathrm{H} \alpha$ images, and compares these with a near-infrared $\mathrm{K}_{\mathrm{S}}$-band image kindly provided by O. Vaduvescu.

The broadband images reveal a smooth stellar component, extending from northeast to southwest, that is $\sim 300^{\prime \prime}(\sim 7.8 \mathrm{kpc}$ at $5.4 \mathrm{Mpc}$ ) in diameter. While the stellar density distribution results in an overall low-surface brightness appearance, the central stellar component is reasonably symmetric and well-defined. We will refer to this $\sim 8 \mathrm{kpc}$ diameter

\footnotetext{
${ }^{1}$ The KPNO 0.9-meter telescope is operated jointly by a consortium that includes the University of Florida, Indiana University, San Francisco State University, Wesleyan University, the University of Wisconsin-Madison, the University of Wisconsin-Oshkosh, the University of Wisconsin-Stevens Point, and the University of Wisconsin-Whitewater
} 
stellar distribution as the central stellar component throughout the rest of the paper. The high foreground contamination from Milky Way stars is clear in the broadband imges. Most of the bright point sources within the disk are foreground stars; the continuum subtraction process (see below) shows that only a few are stellar clusters associated with ongoing SF in the Orion dwarf itself. The most prominent of these clusters are discussed further below.

We use standard techniques to subtract the continuum from the narrowband $\mathrm{H} \alpha$ images. First, the images are cosmic ray rejected, aligned and combined. The broad and narrowband images are then smoothed to a common point spread function size and flux scaled to remove the continuum from the narrowband image. Finally, standard photometry routines are applied, using observations of photometric standard stars acquired during the same observing session. We estimate the photometric accuracy of our final images to be $10 \%$ or better.

Comparing the continuum-subtracted $\mathrm{H} \alpha$ image with the broadband $\mathrm{R}$ and $\mathrm{K}_{\mathrm{S}}$-images in Figure 1, we find that most of the active SF probed by our $\mathrm{H} \alpha$ imaging is contained in moderate-flux H II regions scattered widely throughout the disk. We catalog 18 individual $\mathrm{H}$ II regions, many of which are located well outside of the central stellar component as seen in the $\mathrm{R}$ and $\mathrm{K}_{\mathrm{S}}$-band images. Only three regions (labeled as \#3, 10, 11 in Figure 1) are associated with high surface brightness stellar clusters in the broadband images. We catalog the positions, observed and (Galactic) extinction-corrected fluxes of these H II regions in Table 2. Note that H II region \#11 consists of three distinct peaks that are just barely resolved; the flux in the table is the sum of these three.

From the continuum-subtracted $\mathrm{H} \alpha$ image we derive a total $\mathrm{H} \alpha$ flux (corrected for foreground extinction) of $(113 \pm 1.3) \times 10^{-14} \mathrm{erg} \mathrm{s}^{-1} \mathrm{~cm}^{-2}$. At the adopted distance of $5.4 \mathrm{Mpc}$, this corresponds to a luminosity of $4.0 \times 10^{39} \mathrm{erg} \mathrm{s}^{-1}$. The Orion dwarf is thus a comparatively quiescent and, as shown in the following sections, gas-rich, dwarf galaxy. We note with interest that the $\mathrm{H} \alpha$ luminosity is close to the transition value found by Lee et al. (2009) where $\mathrm{H} \alpha$ emission begins to systematically under-predict the current star formation rate in dwarf galaxies. Using their prescription (which was constructed using a combination of UV and $\mathrm{H} \alpha$ indicators), we derive a current star formation rate of $\sim 0.04 \mathrm{M}_{\odot} \mathrm{yr}^{-1}$; this value is roughly $30 \%$ higher than the value found by a straightforward application of the Kennicutt (1998a) prescription. While the uncertainties are substantial (foreground extinction, adopted distance), the Orion dwarf appears to be in a state of relative quiescence at the present time. 


\section{2. $\quad V L A$ Spectral Line Observations}

H I spectral line imaging of the Orion dwarf galaxy was obtained with the Very Large Array $\left(V L A{ }^{2}\right)$ on 8 March, 2008. These data were acquired under the auspices of the $V L A / V L B A$ Observing for University Classes program. The array was in the C configuration during these observations; the total on-source integration time was 140 minutes. We supplemented these data with dynamically-scheduled observations with the array in the B configuration on 12 January, 2008 (total on-source integration time $=127$ minutes). A total bandwidth of $3.125 \mathrm{MHz}$ was used, with 128 channels separated by $24.4 \mathrm{kHz}\left(5.12 \mathrm{~km} \mathrm{~s}^{-1}\right)$. All reductions were performed using the Astronomical Image Processing System (AIPS) package.

Data handling followed standard procedures, with necessary modifications to account for the array being in a "hybrid" state containing both VLA and EVLA receivers. 12 of 27 antennae were fitted with $E V L A$ receivers during the B configuration observations; in the C configuration there were $13 E V L A$-equipped antennae. While a complete discussion of the intricacies of data handling during the $V L A$ to $E V L A$ transition is beyond the scope of this paper 3 , we briefly note the most significant changes to the "standard" spectral line reduction steps here. First, an alternative channel zero dataset was created that averaged together central channels well separated from the edges of the bandpass. Second, each calibration step is bracketed by a flag/unflag step for all EVLA-EVLA baselines. The combination of these steps allows us to retain the maximum number of baselines from our observations.

Other than the modifications above (and discussed further on the VLA website), the data handling proceeded normally. First, interference and bad data were removed from each dataset; flux, gain and phase calibrations were applied, derived from observations of $0542+498$ (3C147; primary calibrator) and 0532+075 (secondary calibrator) acquired during each observing session. Based on the measured flux density of the phase calibrator from each observing session, we estimate our absolute flux calibration to be accurate at the $\sim 15 \%$ level. The calibrated $u-v$ datasets were then combined and the underlying continuum was removed.

These combined datasets were then imaged and cleaned using the IMAGR task in AIPS. We produce two datacubes, convolved to circular beam sizes of $10^{\prime \prime}$ and $20^{\prime \prime}$. The rms noise levels per $5.2 \mathrm{~km} \mathrm{~s}^{-1}$ channel are 1.51 and $0.55 \mathrm{~K}$ for the $10^{\prime \prime}$ and $20^{\prime \prime}$ cubes, respectively; the corresponding $3 \sigma \mathrm{H}$ I column density sensitivities are $\mathrm{N}_{\mathrm{HI}}=(4.3,1.5) \times 10^{19} \mathrm{~cm}^{-2}$,

\footnotetext{
${ }^{2}$ The National Radio Astronomy Observatory is a facility of the National Science Foundation operated under cooperative agreement by Associated Universities, Inc.

${ }^{3}$ We refer the reader to www.nrao.edu for detailed discussion.
} 
respectively. We explicitly account for the different beam sizes of the dirty and clean maps using residual flux scaling (e.g., Jörsäter \& van Moorsel 1995; Walter et al. 2007).

Moment maps at $10^{\prime \prime}$ and $20^{\prime \prime}$ resolution (representing integrated intensity, velocity, and velocity dispersion) were created from these data cubes using an approach similar to the one described and applied in Cannon et al. (2009). First, the "low-resolution" (20" beam) cube was blanked at the $2 \sigma$ level. Channels containing line emission were then inspected individually; real and spurious emission were differentiated by requiring structures to be present in 3 or more consecutive channels. This edited cube is used to blank both the "low" and "high-resolution" cubes; this assures that the same regions contribute to both final moment maps. The images of integrated H I emission are discussed in detail in $\S 3$,

The total H I flux integral, derived from the $20^{\prime \prime}$ cube, is $50.3 \pm 5.1 \mathrm{Jy} \mathrm{kms}^{-1}$; this is lower than the single-dish flux density of $80.59 \pm 7.72 \mathrm{Jy} \mathrm{km} \mathrm{s}^{-1}$ found by Springob et al. (2005; note that those authors apply a $\sim 8 \%$ correction for $\mathrm{H}$ I self-absorption). This difference likely arises from missing short spacings that provide sensitivity to diffuse structure; further observations with the array in the compact D configuration would be fruitful in this regard. Using these numbers we derive a total H I mass of $(3.5 \pm 0.5) \times 10^{8} \mathrm{M}_{\odot}$; including a $35 \%$ correction for Helium and molecular material, we adopt $(4.7 \pm 0.7) \times 10^{8} \mathrm{M}_{\odot}$ as the total gas mass $\left(\mathrm{M}_{\text {gas }}\right)$ of the Orion dwarf.

During the reduction of the $\mathrm{H}$ I spectral line data, we averaged 78 line-free channels to produce a $1.4 \mathrm{GHz}$ radio continuum image. This image has a beam size of $13.8^{\prime \prime} \times 12.9^{\prime \prime}$ and an RMS noise level of $0.17 \mathrm{mJy}_{\text {beam }}{ }^{-1}$. We do not detect radio continuum emission from the Orion dwarf at this sensitivity level. The $5 \sigma$ upper limit to the global $1.4 \mathrm{GHz}$ radio continuum flux density is derived to be $\mathrm{S}_{1.4 \mathrm{GHz}} \lesssim 0.85 \mathrm{mJy}$. This is consistent with the NVSS non-detection (Condon 1992). The expected thermal radio continuum flux density, based on the (foreground extinction-corrected) $\mathrm{H} \alpha$ flux, is between 0.9-2.0 mJy (depending on the adopted electron temperature; see Caplan \& Deharveng 1986). Examining the continuum emission from the individual H II regions also shows they they are beyond the sensitivity of our L-band continuum image. The most luminous H II region (region \#11) has an $\mathrm{H} \alpha$ flux that would produce a thermal radio continuum flux of $0.28 \mathrm{mJy}$ (assuming $\mathrm{T}_{\mathrm{e}}=10^{4}$ $\mathrm{K}$ ). This is again well below our detection threshold. Thus, even if nonthermal processes dominate the radio continuum flux in the Orion dwarf, it is below our current sensitivity level to study in detail. 


\section{Gaseous, Stellar, and Dark Components}

\subsection{The Neutral Gas Distribution and Dynamics}

$\mathrm{H}$ I emission from the Orion dwarf is detected in 28 channels of our final datacubes, spanning the heliocentric radial velocity range of $301.8-441.0 \mathrm{~km} \mathrm{~s}^{-1}$. Figure 2 shows the individual channel maps of the $20^{\prime \prime}$-resolution datacube. The ordered rotation of the system is prominent, as is a wealth of structure that permeates the ISM. Especially evident is a kinematic break in the distribution and velocity structure of the gas in the central regions of the disk (consider the panels of Figure 2 surrounding $379.2 \mathrm{~km} \mathrm{~s}^{-1}$ ). We explore this structure in more detail below.

Integrating the sum of the flux in each of the channel maps shown in Figure 2 allows us to extract the global H I profile shown in Figure 3. As expected from the rotation of the system evident in Figure 2, we see a very symmetric, double-peaked H I profile. We derive an $\mathrm{H}$ I systemic velocity of $368.5 \pm 1.0 \mathrm{~km} \mathrm{~s}^{-1}$; this velocity is noted in Figure 3 . The full width of the profile at half maximum is $118 \pm 4 \mathrm{~km} \mathrm{~s}^{-1}$; this can be compared with line widths between 97 and $129 \mathrm{~km} \mathrm{~s}^{-1}$ in Springob et al. (2005; the different techniques used to extract the line widths in that work account for the range of derived values).

In Figure 4 we present $\mathrm{H}$ I moment zero (integrated intensity) images of the Orion dwarf at $10^{\prime \prime}$ and $20^{\prime \prime}$ resolutions. As noted for the individual channel maps, the $\mathrm{H}$ I disk shows rich structure at these physical resolutions. The outer disk contains tenuous H I gas, but column densities rise above the $5 \times 10^{20} \mathrm{~cm}^{-2}$ level at intermediate radii. There is plentiful high-column density $\left(>10^{21} \mathrm{~cm}^{-2}\right) \mathrm{H}$ I throughout the disk. It is interesting, however, that the inner regions of the $\mathrm{H}$ I disk are not the regions with the highest gas surface densities. The kinematic features noted in the channel maps are apparently associated with prominent regions of low column density $\left(<3 \times 10^{20} \mathrm{~cm}^{-2}\right)$ in the inner regions. We hereafter refer to these regions as H I "depressions" or "holes" (see further discussion below).

The $\mathrm{H}$ I disk is significantly larger than the central stellar component of the system; Figure 5 compares the optical R-band and $\mathrm{H} \alpha$ images with the $\mathrm{H}$ I distribution (shown by the $10^{21} \mathrm{~cm}^{-2}$ contour). Note that most of the the central stellar component (traced by the R-band image) is located near the $\mathrm{H}$ I depressions. In contrast, most (but not all) of the high surface density $\mathrm{H}$ I gas $\left(\mathrm{N}_{\mathrm{HI}}>10^{21} \mathrm{~cm}^{-2}\right)$ is exterior to the red stellar population.

Figure 5 also compares the locations of high-column density $\mathrm{H}$ I gas and high surface brightness H II regions. We find a strong correlation between $\mathrm{H}$ I gas at or above the $10^{21}$ $\mathrm{cm}^{-2}$ level and regions of active SF as traced by $\mathrm{H} \alpha$ emission. This is further verification of the well-known empirical "star formation threshold" (e.g., Skillman 1987, Kennicutt 1989, 
Kennicutt 1998b, and references therein): high H I column densities are often co-spatial with regions of recent SF. We note that only two of our 18 detected H II regions (specifically, regions 7 and 9 in Table 2, see also Figure 51) are separated by one 10" beam element or more from $\mathrm{H}$ I column densities of $10^{21} \mathrm{~cm}^{-2}$.

The channel maps shown in Figure 2 suggest well-ordered rotation throughout the disk. The first moment image (representing intensity-weighted velocity) presented in Figure 6 confirms this. Isovelocity contours are symmetric in the outer disk, indicative of solid-body rotation. In the central regions of the disk, however, the H I "holes/depressions" manifest a pronounced kink in these contours (consider the contours at $370 \pm 20 \mathrm{~km} \mathrm{~s}^{-1}$ ). This suggests a significant departure from ordered rotation (i.e., non-circular motion) within the inner disk of the Orion dwarf.

In Figure 7 we compare the $\mathrm{H}$ I column density distribution with the second moment image (representing intensity weighted velocity dispersion). Throughout most of the disk, the dispersion is nearly constant at $\sigma_{\mathrm{V}} \simeq 7 \pm 2 \mathrm{~km} \mathrm{~s}^{-1}$. However, this dispersion increases markedly in the inner disk region, corresponding roughly to the location of the central stellar component. There, values reach $\sim 15$ and $20 \mathrm{~km} \mathrm{~s}^{-1}$ in the $20^{\prime \prime}$ and $10^{\prime \prime}$ resolution maps, respectively. While beam smearing effects add uncertainty, we can conclude with confidence that the "depressions/holes" in the inner ISM correlate with areas of higher than average velocity dispersion.

The proximity of this galaxy, and the well-ordered rotation throughout most of the outer $\mathrm{H}$ I disk, are conducive to rotation curve analysis. To this end, we fitted tilted ring models to the velocity field using tools in the GIPSY software package 4 . In an iterative sequence, we fit the systemic velocity $\left(\mathrm{V}_{\text {sys }}\right)$, dynamical center position, position angle (P.A.), inclination (i) and rotational velocity for the galaxy as a whole, and for the receding and approaching sides individually. Optimal fits were obtained with $i=(45 \pm 3)^{\circ}$, P.A. $=(20 \pm 2)^{\circ}, \mathrm{V}_{\text {sys }}=$ $(368.5 \pm 1.0) \mathrm{km} \mathrm{s}^{-1}$, and a dynamical center at $(\alpha, \delta, \mathrm{J} 2000)=(05: 45: 01.66,05: 03: 55.2)$. The resulting curves, shown at both $10^{\prime \prime}$ and $20^{\prime \prime}$ resolution in Figure 8, demonstrate solid-body rotation to $\sim 140^{\prime \prime}(3.7 \mathrm{kpc})$ and a flattening at $\sim 82 \mathrm{~km} \mathrm{~s}^{-1}$ to the detection limit in the outer disk $(\sim 6.8 \mathrm{kpc})$. Assuming circular orbits, the implied dynamical mass at this radius is $10.6 \times 10^{9} \mathrm{M}_{\odot}$.

Our tilted ring analysis also allows us to examine the radial behavior of $\mathrm{H}$ I mass density per unit area throughout the gas disk. We again used the GIPSY software package to integrate the $\mathrm{H}$ I flux per unit area, in concentric rings separated by the beam widths,

\footnotetext{
${ }^{4}$ The Groningen Image Processing System (GIPSY) is distributed by the Kapteyn Atronomical Institute, Groningen, Netherlands.
} 
after correcting for inclination and the galaxy's major axis position angle. The resulting plot, shown in Figure 9, clearly demonstrates that the inner region of the H I disk where the "depressions/holes" are located contains significantly less neutral gas per unit area than the regions further out in the disk. The highest mass surface densities are located between $\sim 1.5-3 \mathrm{kpc}$ from the dynamical center; the inner disk has a mass surface density $\sim 40 \%$ lower at these physical resolutions.

\subsection{The Old and Young Stellar Populations}

As noted above, the dynamical mass exceeds the gaseous mass (even when accounting for missing $\mathrm{H}$ I flux compared to single-dish observations). We now explore what fraction of this dynamical mass is in the form of stars. The underlying stellar mass is estimated using the near-infrared (IR) photometry $\left(\mathrm{J}\right.$ and $\mathrm{K}_{\mathrm{S}}$ bands) presented by Vaduvescu et al. (2005). Those authors find $\left(\mathrm{J}-\mathrm{K}_{\mathrm{S}}\right)=+0.80$ and a total $\mathrm{K}_{\mathrm{S}}$ magnitude of +10.90 . When comparing to models (see below) we assume that the color difference between $\mathrm{K}$ and $\mathrm{K}_{\mathrm{S}}$ is negligible; further, we assume $\mathrm{L}_{\mathrm{K}, \odot}=+3.33$ (Cox 2000; Bessell 1979). Accounting for extinction (see above), the total K-band luminosity of the Orion dwarf is $\sim 3.5 \times 10^{8} \mathrm{~L}_{\odot}$.

To estimate the mass of the stellar component, we apply the models presented in Bell \& de Jong (2001). Using an observed color, one can infer a stellar mass-to-light ratio (M/L) for a variety of stellar population synthesis models that cover a range of metallicities, initial mass functions, and galaxy evolution properties. The near-infrared colors are least susceptible to variations due to internal extinction or recent SF. In order to compare the near-IR photometry with the Bell \& de Jong (2001) models, we followed the approach discussed in Lee et al. (2006) and derived a general relation between ( $\mathrm{J}-\mathrm{K}$ ) color and the stellar M/L ratio as a linear combination of $(\mathrm{V}-\mathrm{J})$ and $(\mathrm{V}-\mathrm{K})$ indices. Using the sub-solar metallicity models in Bell \& de Jong (2001), this yields estimates of the stellar M/L ratio for each model. Using these M/L ratios and the observed K-band luminosity, we then obtain estimates of the galaxy's stellar mass. Using the dispersion amongst the low-metallicity models as an indicator of the accuracy of this method, we conclude that the underlying stellar mass in the Orion dwarf is $(3.7 \pm 1.5) \times 10^{8} \mathrm{M}_{\odot}$. A similar calculation that uses all

available models in the Bell \& de Jong (2001) work (i.e., including those for all metallicities) finds a similar (equal within errors), but slightly lower, value of $(2.9 \pm 1.3) \times 10^{8} \mathrm{M}_{\odot}$.

This stellar mass is comparable to, but on the high end of, the distribution of stellar masses in local dwarfs found by Lee et al. (2006) While that investigation uses a longer color baseline, our techniques for deriving the stellar masses are identical. The Orion dwarf has a stellar mass comparable to that of the well-studied irregulars NGC 4214 and NGC 55; its 
current ( $\mathrm{H} \alpha$-derived) star formation rate is lower, however, than in those systems.

\section{3. $\quad$ A Dark Matter Dominated Galaxy}

Like most dwarf galaxies, the Orion dwarf appears to be dark-matter dominated throughout the disk. In $\S 2.2$ we derived a total gas mass $\mathrm{M}_{\text {gas }}=(4.7 \pm 0.7) \times 10^{8} \mathrm{M}_{\odot}$; this includes a $35 \%$ correction for Helium and molecular material. From $\S 3.2$, the total stellar mass $M_{\star}$ $=(3.7 \pm 1.5) \times 10^{8} \mathrm{M}_{\odot}$. The resulting mass ratio of gas to stars, $\log \left(\mathrm{M}_{\text {gas }} / \mathrm{M}_{\star}\right) \simeq 0.10$, is comparable to many systems in the Lee et al. (2006) study.

The masses of these luminous components can be compared with the total dynamical mass of the system (from $\S 3.1, \mathrm{M}_{\mathrm{dyn}} \simeq 10.6 \times 10^{9} \mathrm{M}_{\odot}$ ). This implies a $\mathrm{M} / \mathrm{L}$ ratio of $\sim 13$ for the galaxy as a whole. Continuing inward through the disk the M/L ratio remains above unity; most of the $\mathrm{K}_{\mathrm{S}}$-band luminosity is contained within $\sim 3^{\prime}$ of the dynamical center (see, for example, Figures 1 and 5). At the point where the rotation curve flattens $\left(\simeq 140^{\prime \prime}=\right.$ $3.4 \mathrm{kpc}$ ) the rotation velocity is $\sim 79 \mathrm{~km} \mathrm{~s}^{-1}$; the interior dynamical mass is $\sim 5.3 \times 10^{9} \mathrm{M}_{\odot}$, already exceeding the total luminous mass in the entire galaxy.

\section{The Star Formation - ISM Connection}

The rich ISM structure seen in the integrated H I distribution (see Figure 4), and the locations of the stellar distribution and the sites of recent SF within the disk (see Figure 5), are suggestive of a formative link between recent SF and the observed structures of the ISM. As noted in $\S$ 3.1, the most prominent kinematic features are apparent in both the individual channel maps (see Figure 2) and in the integrated distribution (see Figure 5) as regions of low column density. We now explore the nature of the three most prominent of these "depressions/holes" in more detail.

To better elucidate the positions and morphologies of these features, we overlay their positions on the $\mathrm{H}$ I integrated intensity map, the $\mathrm{H}$ I velocity field, and the $\mathrm{H}$ I velocity dispersion map in Figure 10, We number these features in order of increasing right ascension and summarize their basic properties in Table 3. The "depressions/holes" are spatially coincident with departures from ordered isovelocity contours, have lower than average H I column density, and have higher than average $\mathrm{H}$ I velocity dispersion.

Figure 11] shows these same ellipses overlaid on optical R-band and continuum-subtracted $\mathrm{H} \alpha$ images; the field of view is the same as in Figure 5. Each of the kinematic features is completely enclosed within the $\mathrm{N}_{\mathrm{HI}}=10^{21} \mathrm{~cm}^{-2}$ contour. None of them is cospatial with 
the central stellar disk; each is offset by some amount, with feature \#3 showing the least positional agreement with the stellar distribution.

When comparing the locations of these features with the individual channel maps, however, "depression/hole" \#3 (which shows the poorest positional agreement with the stellar distribution) is the easiest to identify. Examining Figure 12, which shows the same channel maps as Figure 2 but now overlaid with the locations of the "depressions/holes", this feature is clearly defined as a dearth of $\mathrm{H}$ I emission in the panels near $379 \mathrm{~km} \mathrm{~s}^{-1}$. As expected, this feature causes non-uniformities in the integrated velocity field; Figure 10 shows this quite clearly.

If these features are in fact holes in the ISM, it is natural to search for kinematic verification of their expansion. To this end, we searched the datacubes for kinematic signatures of expansion of the major observed H I structures. We examined both position-velocity and radius-velocity diagrams throughout the $\mathrm{H}$ I disk. These features are each detected as pronounced breaks in the local $\mathrm{H}$ I distribution as seen in position-velocity space. We show one such profile in Figure 13, in both major- and minor-axis cuts, the "depression/hole" features are clearly evident as kinematic discontinuities in the inner disk.

While the position-velocity diagrams verify the lower-than-average column densities of these features, radius-velocity diagrams do not find unambiguous evidence for expansion of these structures at the present time. If these features are expanding within the disk, they must be doing so at a modest rate $\left(\mathrm{V}_{\exp } \lesssim 10 \mathrm{~km} \mathrm{~s}^{-1}\right.$, or roughly two channel widths). We note that significant expansion may go undetected within these data if that motion is significantly offset with respect to the galaxy's disk.

These types of features in the ISM of nearby galaxies are often attributed to stellar evolution processes (i.e., stellar winds and supernovae). In the simplest sense, these processes deposit energy into the ISM of the host galaxy; this energy evacuates the gas surrounding regions of strong recent SF. The "simplistic" nature of dwarfs is advantageous in this regard, as such features may be comparatively long-lived. This "feedback" mechanism thus provides a direct link between the stellar and gaseous components of a galaxy.

Recent work by Weisz etal. (2009) has suggested that stellar evolution processes are energetically capable of producing adequate energies to drive expanding shells in the ISM of low-mass galaxies. An important step toward understanding this mechanism was taken by other works that suggest that these stellar evolution processes need not necessarily be "instantaneous". Rather, recent observations have revealed that periods of elevated star formation rates can be sustained in low-mass galaxies for hundreds of Myr (see Cannon et al. 2003, Weisz et al. 2008, Mc Quinn et al. 2009). Such events can inject large energies into the 
ISM without the requisite of leaving a remnant stellar cluster (signifying an instantaneous burst) behind.

Unfortunately we are unable to directly test this model with our present H I imaging of the Orion dwarf. An estimate of the energetic requirements to evacuate these features is very difficult without a measurement of the present expansion rate. To accurately infer the energetic requirements, one would ideally model the underlying mass distribution using deep infrared imaging (Oh et al. 2008) and use this, in combination with the H I velocity dispersion, to derive the $\mathrm{H}$ I scale height as a function of radius. These values can then be used to estimate the $\mathrm{H}$ I number density in the gas prior to the creation of the structure. The energetic requirement is then straightforward: move a known quantity of gas by a measured amount in a given amount of time (based on the "kinematic age" of the structure, which depends on its expansion velocity). To draw definitive conclusions on the "feedback" scenario, one also must have deep, single-star photometry in order to reconstruct the recent star formation history (see Weisz et al. 2008 and 2009). Unfortunately, the large foreground extinction toward the Orion dwarf makes such an endeavor very challenging.

If the observed structures are in fact the products of stellar evolution, then, adopting the formalism of Brinks \& Bajaja (1986) and Walter \& Brinks (1999), these structures may be classified as regions of "total blowout". Any hole or shell structures have either stalled completely (meaning they have reached pressure equilibrium with the surrounding ISM or have broken out perpendicular to the disk) or were sufficiently extended (spatially or temporally) so as to preclude the formation of a single observable kinematic feature. Such a scenario appears increasingly reasonable based on recent studies of the star formation histories of low-mass galaxies (Weisz et al. 2008 and 2009, Mc Quinn et al. 2009), but we must await further data to study this hypothesis in the Orion dwarf.

We note that various alternatives to the stellar evolution model have been postulated (see, for example, the discussion in Rhode et al. 1999). Given the number of assumptions required to derive the creation energy of an expanding structure from observations, it is not surprising that one of the leading alternatives is simply that the energetics are incorrectly attained. We note that the methods for deriving these numbers are using increasing levels of sophistication (e.g., Oh et al. 2008). Similarly, spatially resolved stellar population work is helping to eliminate non-standard initial mass functions as the explanation. Other hypotheses are more challenging to disregard and they remain theoretically viable. Some of these include high velocity cloud impacts, disk instabilities, turbulence, or ram pressure stripping (e.g., Sánchez-Salcedo 2002). 


\section{Conclusions}

New H I and optical imaging of the Orion dwarf have been presented. The stellar component occupies the inner region of a mostly well-ordered $\mathrm{H}$ I disk. Column densities rise above the $10^{21} \mathrm{~cm}^{-2}$ level in multiple areas, although the central stellar component is primarily coincident with lower surface density gas in the inner disk. This area contains three regions of lower than average column density, higher than average velocity dispersion, and departures from symmetric isovelocity contours; we term these features "holes/depressions". The areas of active SF as traced by $\mathrm{H} \alpha$ emission show a strong preference for high surface density gas; some of the active regions are well outside of the central stellar component of the system.

The Orion dwarf is well-suited for rotation curve analysis. Through an iterative procedure we extract a rotation curve that is essentially flat at $\sim 82 \mathrm{~km} \mathrm{~s}^{-1}$ out to the edge of the $\mathrm{H}$ I disk $(\simeq 6.8 \mathrm{kpc})$. At the last rotation curve point, the implied dynamical mass $\mathrm{M}_{\text {dyn }} \simeq 10.6 \times 10^{9} \mathrm{M}_{\odot}$. Applying the models of Bell \& de Jong (2001), we use optical and infrared images to derive a stellar mass of $(3.7 \pm 1.5) \times 10^{8} \mathrm{M}_{\odot}$. This can be compared with the neutral gas mass of $(4.7 \pm 1.5) \times 10^{8} \mathrm{M}_{\odot}$ (which includes a correction for helium and molecular material). The Orion dwarf is thus dark matter dominated throughout the disk.

We investigate the kinematics of the "hole/depression" regions. While these appear as prominent breaks in position-velocity space, and are evident in individual channel maps, a radius-velocity analysis does not show signs of expansion of these features at the present sensitivity and velocity resolution. Unfortunately, this lack of a measured expansion velocity for these features precludes a direct derivation of the energetic requirements for their formation.

While the evidence for "feedback" (i.e., the effects of energy released by stellar evolution processes on the surrounding ISM) is tantalizing, we are unable to directly test this

hypothesis. In addition to higher velocity resolution H I imaging (necessary to measure a potentially slower expansion rate for the "hole/depression" structures), we also require deep, single-star photometry to unambiguously connect the past SF with the current ISM features. The high foreground reddening toward the Orion dwarf will make this challenging; however, the curious properties of this system suggest that the investment will be a worthwhile one in the quest to connect SF activity with characteristics of the ISM in dwarf galaxies.

The authors would like to thank the NRAO for making the VLA/VLBA Observing for University Classes Program available to the educational community, and for travel support and hospitality while at the Array Operations Center. We would also like to thank Robert Dickman, Vivek Dhawan, and the AOC staff for organizing a most productive and enjoyable 
visit. Thanks to Ovidiu Vaduvescu for allowing us to analyze and present the infrared image in this manuscript. We acknowledge a helpful anonymous referee whose comments improved this manuscript. JMC thanks Macalester College for research and teaching support that made this project possible, and thanks Henry Lee for helpful discussions. The optical observations were made possible through financial support from Indiana University and a grant from the National Science Foundation (AST-0823801) to JJS. This research has made use of the NASA/IPAC Extragalactic Database (NED) which is operated by the Jet Propulsion Laboratory, California Institute of Technology, under contract with the National Aeronautics and Space Administration, and NASA's Astrophysics Data System. 


\section{REFERENCES}

Bell, E. F., \& de Jong, R. S. 2001, ApJ, 550, 21

Bessell, M. S. 1979, PASP, 91, 589

Brinks, E., \& Bajaja, E. 1986, A\&A, 169, 14

Cannon, J. M., Dohm-Palmer, R. C., Skillman, E. D., Bomans, D. J., Côté, S., \& Miller, B. W. 2003, AJ, 126, 2806

Cannon, J. M., Salzer, J. J., \& Rosenberg, J. L. 2009, ApJ, 696, 2104

Condon, J. J. 1992, ARA\&A, 30, 575

Cox, A. N. 2000, Allen's Astrophysical Quantities (4th ed.; New York; Springer)

Caplan, J., \& Deharveng, L. 1986, A\&A, 155, 297

Giovanelli, R. 1979, ApJ, 227, L125

Hunter, D. A., Brinks, E., Elmegreen, B., Rupen, M., Simpson, C., Walter, F., Westpfahl, D., \& Young, L. 2007, Bulletin of the American Astronomical Society, 38, 895

Jörsäter, S., \& van Moorsel, G. A. 1995, AJ, 110, 2037

Lee, H., Skillman, E. D., Cannon, J. M., Jackson, D. C., Gehrz, R. D., Polomski, E. F., \& Woodward, C. E. 2006, ApJ, 647, 970

Lee, J. C., et al. 2009, ApJ, 706, 599

Karachentsev, I., \& Musella, I. 1996, A\&A, 315, 348

Kennicutt, R. C., Jr. 1989, ApJ, 344, 685

Kennicutt, R. C., Jr. 1998a, ARA\&A, 36, 189

Kennicutt, R. C., Jr. 1998b, ApJ, 498, 541

Kim, S., Dopita, M. A., Staveley-Smith, L., \& Bessell, M. S. 1999, AJ, 118, 2797

Mateo, M. L. 1998, ARA\&A, 36, 435

McQuinn, K. B. W., Skillman, E. D., Cannon, J. M., Dalcanton, J. J., Dolphin, A., Stark, D., \& Weisz, D. 2009, ApJ, 695, 561 
Oh, S.-H., de Blok, W. J. G., Walter, F., Brinks, E., \& Kennicutt, R. C. 2008, AJ, 136, 2761

Ott, J., Walter, F., Brinks, E., Van Dyk, S. D., Dirsch, B., \& Klein, U. 2001, AJ, 122, 3070

Puche, D., Westpfahl, D., Brinks, E., \& Roy, J.-R. 1992, AJ, 103, 1841

Puche, D., \& Westpfahl, D. 1994, European Southern Observatory Astrophysics Symposia, 49,273

Rhode, K. L., Salzer, J. J., Westpfahl, D. J., \& Radice, L. A. 1999, AJ, 118, 323

Sánchez-Salcedo, F. J. 2002, Revista Mexicana de Astronomia y Astrofisica, 38, 39

Schlegel, D. J., Finkbeiner, D. P., \& Davis, M. 1998, ApJ, 500, 525

Skillman, E. D. 1987, NASA Conference Publication, 2466, 263

Springob, C. M., Haynes, M. P., Giovanelli, R., \& Kent, B. R. 2005, ApJS, 160, 149

Tenorio-Tagle, G., \& Bodenheimer, P. 1988, ARA\&A, 26, 145

Vaduvescu, O., McCall, M. L., Richer, M. G., \& Fingerhut, R. L. 2005, AJ, 130, 1593

Walter, F., \& Brinks, E. 1999, AJ, 118, 273

Walter, F., \& Brinks, E. 2001, AJ, 121, 3026

Walter, F., et al. 2007, ApJ, 661, 102

Walter, F., Brinks, E., de Blok, W. J. G., Bigiel, F., Kennicutt, R. C., Thornley, M. D., \& Leroy, A. 2008, AJ, 136, 2563

Weisz, D. R., Skillman, E. D., Cannon, J. M., Dolphin, A. E., Kennicutt, R. C., Jr., Lee, J., \& Walter, F. 2008, ApJ, 689, 160

Weisz, D. R., Skillman, E. D., Cannon, J. M., Dolphin, A. E., Kennicutt, R. C., Lee, J., \& Walter, F. 2009, ApJ, 704, 1538

Weldrake, D. T. F., de Blok, W. J. G., \& Walter, F. 2003, MNRAS, 340, 12 
Table 1. Basic Characteristics of the Orion Dwarf Galaxy

\begin{tabular}{|c|c|}
\hline Parameter & Value \\
\hline Right ascension (J2000) & $05^{\mathrm{h}} 45^{\mathrm{m}} 01 .{ }^{\mathrm{s}} 6$ \\
\hline Declination (J2000) & $+05^{\circ} 03^{\prime} 41^{\prime \prime}$ \\
\hline Adopted distance (Mpc) & $5.4^{\mathrm{a}}$ \\
\hline$A_{R}$ & $1.959^{\mathrm{b}}$ \\
\hline Interferometric $\mathrm{S}_{\mathrm{HI}}(\mathrm{Jy} \mathrm{km} / \mathrm{s})$ & $50.3 \pm 5.1$ \\
\hline Single-dish $\mathrm{S}_{\mathrm{HI}}(\mathrm{Jy} \mathrm{km} / \mathrm{s})$ & $80.59 \pm 7.72^{\mathrm{c}}$ \\
\hline H I mass $\mathrm{M}_{\mathrm{HI}}\left(\mathrm{M}_{\odot}\right)$ & $(3.46 \pm 0.5) \times 10^{8}$ \\
\hline \multicolumn{2}{|l|}{ Vaduvescu et al. (2005) } \\
\hline \multicolumn{2}{|l|}{ bchlegel et al. (1998) } \\
\hline Springob et al. (2005) & \\
\hline
\end{tabular}


Table 2. $\mathrm{H} \alpha$ Emission Line Region Catalog

\begin{tabular}{lcccc}
\hline \hline Number & $\begin{array}{c}\alpha^{\mathrm{a}} \\
(\mathrm{J} 2000)\end{array}$ & $\begin{array}{c}\delta^{\mathrm{a}} \\
(\mathrm{J} 2000)\end{array}$ & $\begin{array}{c}\text { Observed H } \alpha \text { Flux } \\
\left(10^{-14} \mathrm{erg} \mathrm{s}^{-1} \mathrm{~cm}^{-2}\right)\end{array}$ & $\begin{array}{c}\text { Extinction-Corrected H } \alpha \text { Flux } \\
\left(10^{-14} \mathrm{erg} \mathrm{s}^{-1} \mathrm{~cm}^{-2}\right)\end{array}$ \\
\hline 1 & $05^{\mathrm{h}} 44^{\mathrm{m}} 56.47^{\mathrm{s}}$ & $05^{\circ} 02^{\prime} 44.44^{\prime \prime}$ & $0.57 \pm 0.06$ & $3.46 \pm 0.36$ \\
2 & $05^{\mathrm{h}} 44^{\mathrm{m}} 57.95^{\mathrm{s}}$ & $05^{\circ} 03^{\prime} 20.11^{\prime \prime}$ & $0.64 \pm 0.04$ & $3.89 \pm 0.24$ \\
3 & $05^{\mathrm{h}} 44^{\mathrm{m}} 59.70^{\mathrm{s}}$ & $05^{\circ} 04^{\prime} 03.90^{\prime \prime}$ & $0.98 \pm 0.04$ & $5.95 \pm 0.24$ \\
4 & $05^{\mathrm{h}} 44^{\mathrm{m}} 59.99^{\mathrm{s}}$ & $05^{\circ} 03^{\prime} 25.04^{\prime \prime}$ & $2.05 \pm 0.06$ & $12.45 \pm 0.36$ \\
5 & $05^{\mathrm{h}} 45^{\mathrm{m}} 00.39^{\mathrm{s}}$ & $05^{\circ} 03^{\prime} 03.75^{\prime \prime}$ & $1.24 \pm 0.05$ & $7.53 \pm 0.30$ \\
6 & $05^{\mathrm{h}} 45^{\mathrm{m}} 00.44^{\mathrm{s}}$ & $05^{\circ} 03^{\prime} 15.33^{\prime \prime}$ & $0.60 \pm 0.04$ & $3.64 \pm 0.24$ \\
7 & $05^{\mathrm{h}} 45^{\mathrm{m}} 00.87^{\mathrm{s}}$ & $05^{\circ} 05^{\prime} 38.32^{\prime \prime}$ & $0.23 \pm 0.03$ & $1.40 \pm 0.18$ \\
8 & $05^{\mathrm{h}} 45^{\mathrm{m}} 00.99^{\mathrm{s}}$ & $05^{\circ} 03^{\prime} 15.88^{\prime \prime}$ & $1.01 \pm 0.05$ & $4.13 \pm 0.30$ \\
9 & $05^{\mathrm{h}} 45^{\mathrm{m}} 01.68^{\mathrm{s}}$ & $05^{\circ} 02^{\prime} 44.11^{\prime \prime}$ & $0.82 \pm 0.04$ & $13.66 \pm 0.36$ \\
10 & $05^{\mathrm{h}} 45^{\mathrm{m}} 01.94^{\mathrm{s}}$ & $05^{\circ} 04^{\prime} 07.87^{\prime \prime}$ & $2.25 \pm 0.06$ & $23.81 \pm 0.55$ \\
11 & $05^{\mathrm{h}} 45^{\mathrm{m}} 02.61^{\mathrm{s}}$ & $05^{\circ} 04^{\prime} 19.13^{\prime \prime}$ & $3.92 \pm 0.09$ & $4.13 \pm 0.36$ \\
12 & $05^{\mathrm{h}} 45^{\mathrm{m}} 02.69^{\mathrm{s}}$ & $05^{\circ} 02^{\prime} 40.95^{\prime \prime}$ & $0.68 \pm 0.06$ & $1.52 \pm 0.18$ \\
13 & $05^{\mathrm{h}} 45^{\mathrm{m}} 03.11^{\mathrm{s}}$ & $05^{\circ} 04^{\prime} 39.36^{\prime \prime}$ & $0.66 \pm 0.04$ & $1.82 \pm 0.30$ \\
14 & $05^{\mathrm{h}} 45^{\mathrm{m}} 03.21^{\mathrm{s}}$ & $05^{\circ} 05^{\prime} 16.82^{\prime \prime}$ & $0.25 \pm 0.03$ & $5.41 \pm 0.30$ \\
15 & $05^{\mathrm{h}} 45^{\mathrm{m}} 03.95^{\mathrm{s}}$ & $05^{\circ} 02^{\prime} 41.47^{\prime \prime}$ & $0.30 \pm 0.05$ & $4.07 \pm 0.18$ \\
16 & $05^{\mathrm{h}} 45^{\mathrm{m}} 04.26^{\mathrm{s}}$ & $05^{\circ} 04^{\prime} 48.27^{\prime \prime}$ & $0.89 \pm 0.05$ & $5.53 \pm 0.36$ \\
17 & $05^{\mathrm{h}} 45^{\mathrm{m}} 04.59^{\mathrm{s}}$ & $05^{\circ} 05^{\prime} 02.30^{\prime \prime}$ & $0.67 \pm 0.03$ & \\
18 & $05^{\mathrm{h}} 45^{\mathrm{m}} 05.53^{\mathrm{s}}$ & $05^{\circ} 03^{\prime} 25.42^{\prime \prime}$ & $0.91 \pm 0.06$ & \\
\hline
\end{tabular}

${ }^{a}$ The right ascension and declination at the estimated central position of the $\mathrm{H} \alpha$ region; estimated astrometric uncertainty is $\pm 0.5^{\prime \prime}$.

${ }^{\mathrm{b}} \mathrm{The} \mathrm{H} \alpha$ fluxes are corrected for the R-band extinction level of 1.959 magnitudes (Schlegel et al. 1998). 


\section{Table 3. Prominent H I "Holes/Depressions" in the Orion Dwarf Galaxy}

\begin{tabular}{lccccc}
\hline \hline Number & $\begin{array}{c}\alpha \\
(\mathrm{J} 2000)^{\mathrm{a}}\end{array}$ & $\begin{array}{c}\delta \\
(\mathrm{J} 2000)^{\mathrm{a}}\end{array}$ & $\begin{array}{c}\text { Major Axis } \\
(\mathrm{pc})^{\mathrm{b}}\end{array}$ & $\begin{array}{c}\text { Minor Axis } \\
(\mathrm{pc})^{\mathrm{b}}\end{array}$ & $\begin{array}{c}\text { Position Angle } \\
(\text { degrees })^{\mathrm{c}}\end{array}$ \\
\hline 1 & $05^{\mathrm{h}} 45^{\mathrm{m}} 00.76^{\mathrm{s}}$ & $05^{\circ} 04^{\prime} 30.5^{\prime \prime}$ & 920 & 650 & 160 \\
2 & $05^{\mathrm{h}} 45^{\mathrm{m}} 02.47^{\mathrm{s}}$ & $05^{\circ} 03^{\prime} 19.0^{\prime \prime}$ & 1440 & 770 & 150 \\
3 & $05^{\mathrm{h}} 45^{\mathrm{m}} 04.45^{\mathrm{s}}$ & $05^{\circ} 04^{\prime} 05.0^{\prime \prime}$ & 1700 & 920 & 40 \\
\hline
\end{tabular}

aThe right ascension and declination of the center of the structure; estimated astrometric uncertainty is $\pm 3^{\prime \prime}$. The locations are shown in panels $c$ and $d$ of Figure 5

${ }^{\mathrm{b}}$ The major and minor axis diameters in pc, using the assumed distance of $5.4 \mathrm{Mpc}$.

${ }^{\mathrm{c}}$ Position angle of the major axis, measured as positive moving eastward from north. 


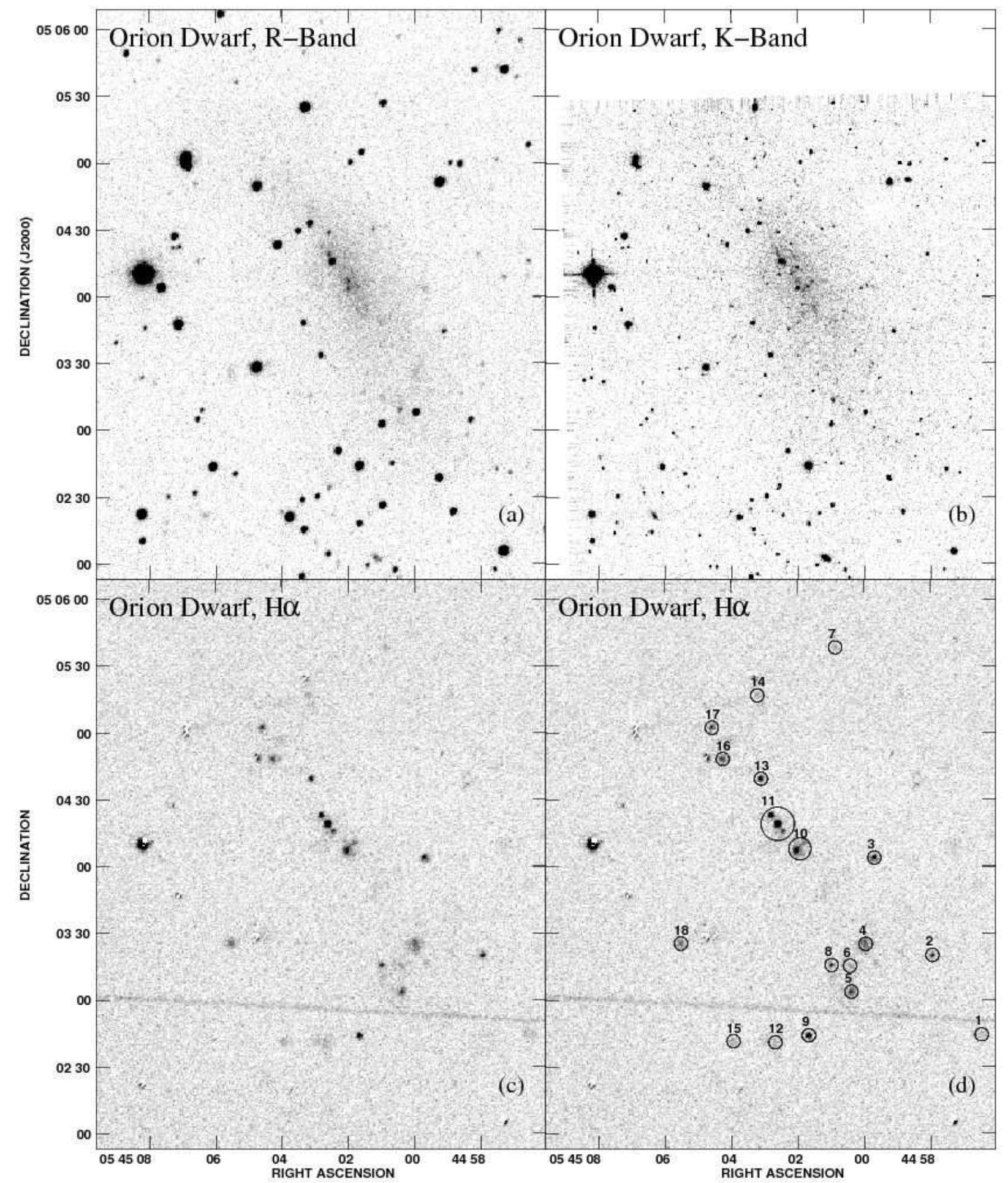

Fig. 1.- Optical and infrared images of the Orion dwarf. Panel $a$ shows the R-band image from the KPNO $0.9 \mathrm{~m}$ telescope; panel $b$ shows the $\mathrm{K}_{\mathrm{S}}$ band image (courtesy of $\mathrm{O}$. Vaduvescu); panel $c$ shows the continuum-subtracted $\mathrm{H} \alpha$ image from the KPNO $0.9 \mathrm{~m}$ telescope; panel $d$ shows the same image as in panel $c$, but with the 18 individual $\mathrm{H}$ II regions numbered in order of increasing Right Ascension (note that Region \#11 consists of three distinct peaks). 


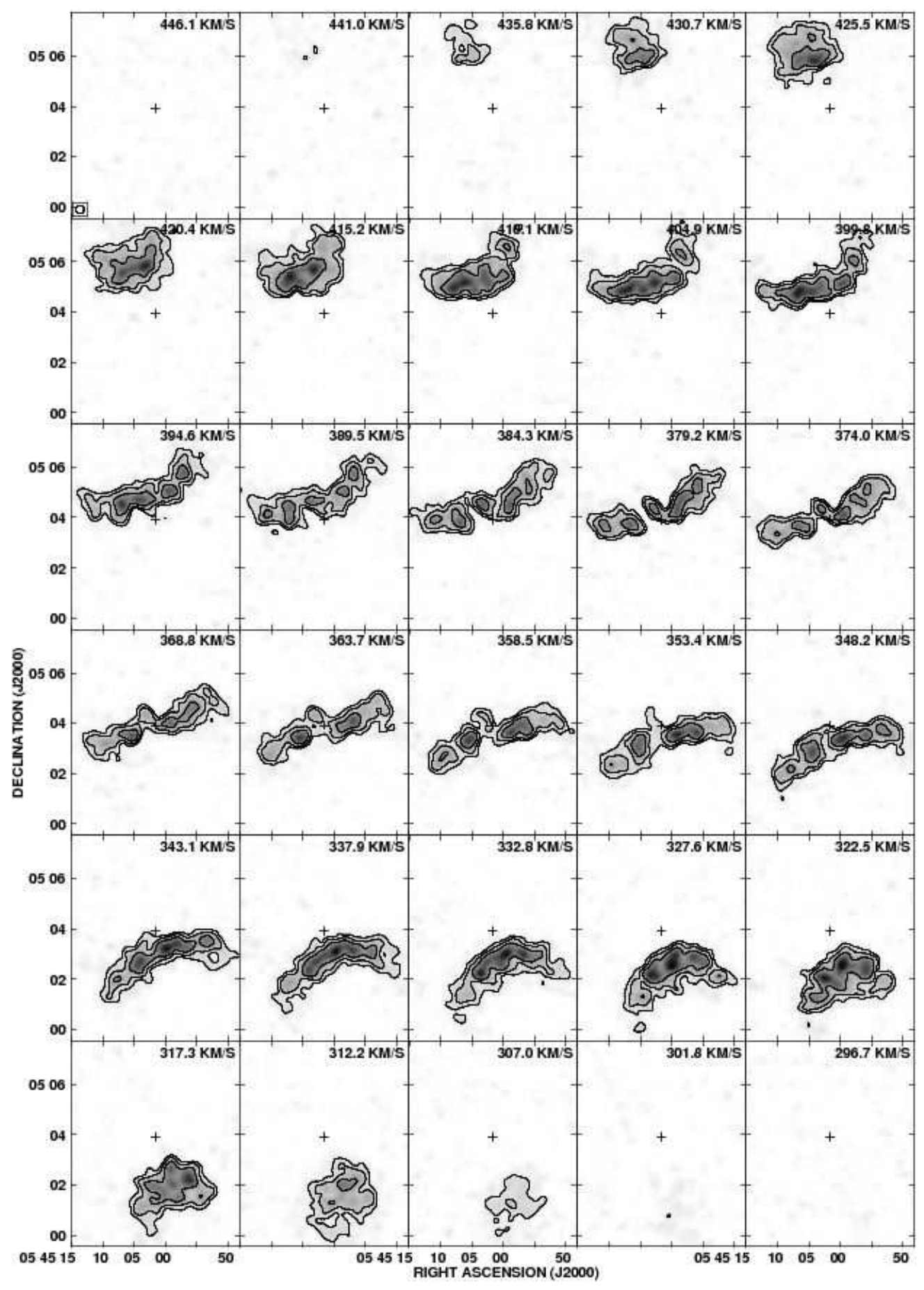

Fig. 2.- Channel maps from the $20^{\prime \prime}$ resolution datacube; heliocentric velocity is noted in the upper right of each frame, and the beam size is shown in the top left panel. Contours are at levels of 2.5, 5, $10 \mathrm{mJy} \mathrm{Bm}^{-1}$. Note the pronounced kinematic break in the central regions (consider the $379.2 \mathrm{~km} \mathrm{~s}^{-1}$ panel and its neighbors). The + sign marks the dynamical center as derived from our tilted ring model fits (see $\S 3.1$ ). 


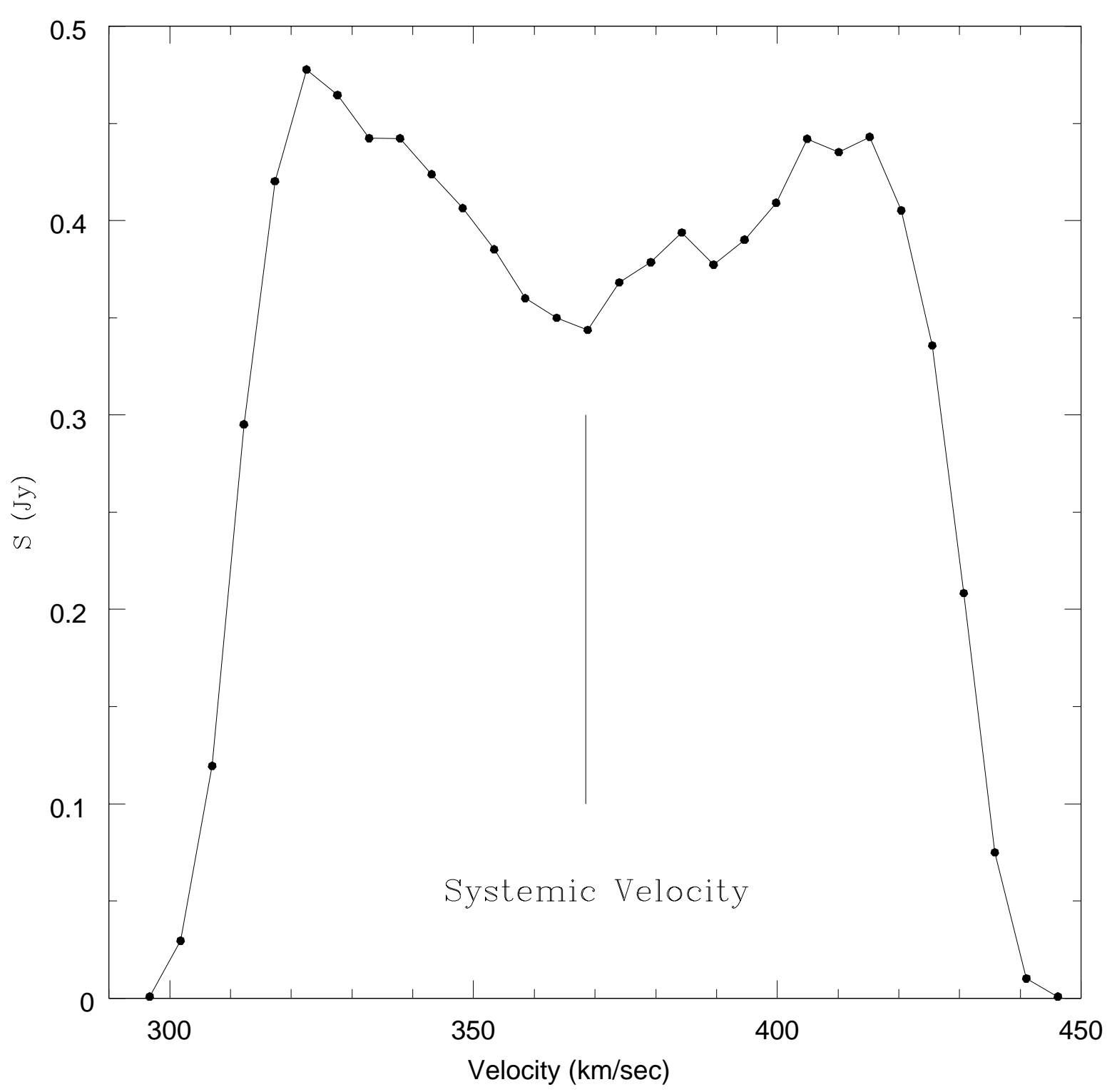

Fig. 3.- The global H I profile of the Orion dwarf, created by summing the flux in each channel of the low-resolution ( $20^{\prime \prime}$ beam), blanked data cube. The systemic velocity, derived from this profile and from our tilted ring analyses (see $\S 3.1$ ), is $368.5 \pm 1.0 \mathrm{~km} \mathrm{~s}^{-1}$. 


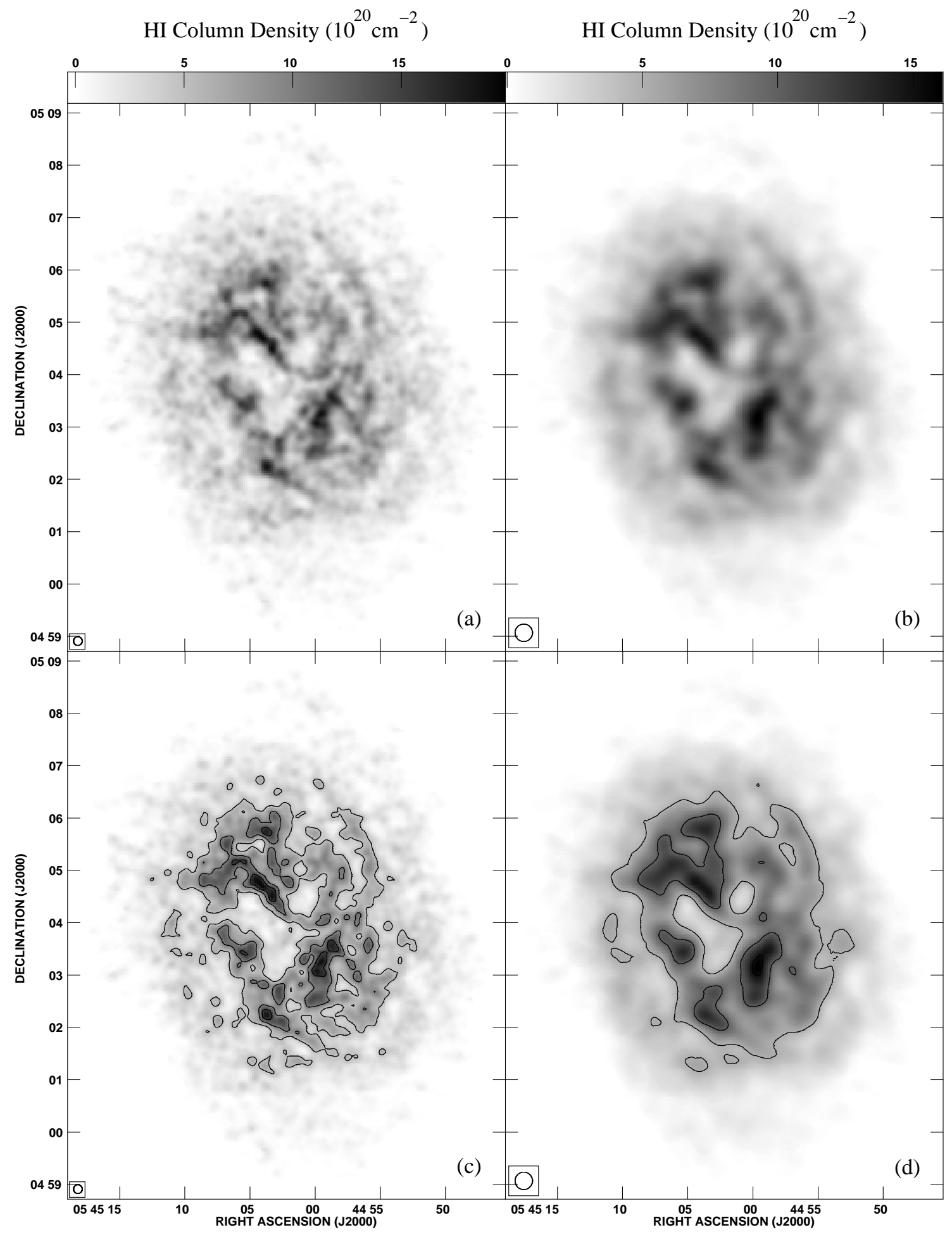

Fig. 4. - The H I column density distribution of the Orion dwarf galaxy at $10^{\prime \prime}$ (panels $a$, c) and $20^{\prime \prime}$ (panels $b, d$ ) resolution. Contours in the lower panels are shown at the $(5,10$, 15) $\times 10^{20} \mathrm{~cm}^{-2}$ levels. A wealth of structure is prominent throughout the disk. 


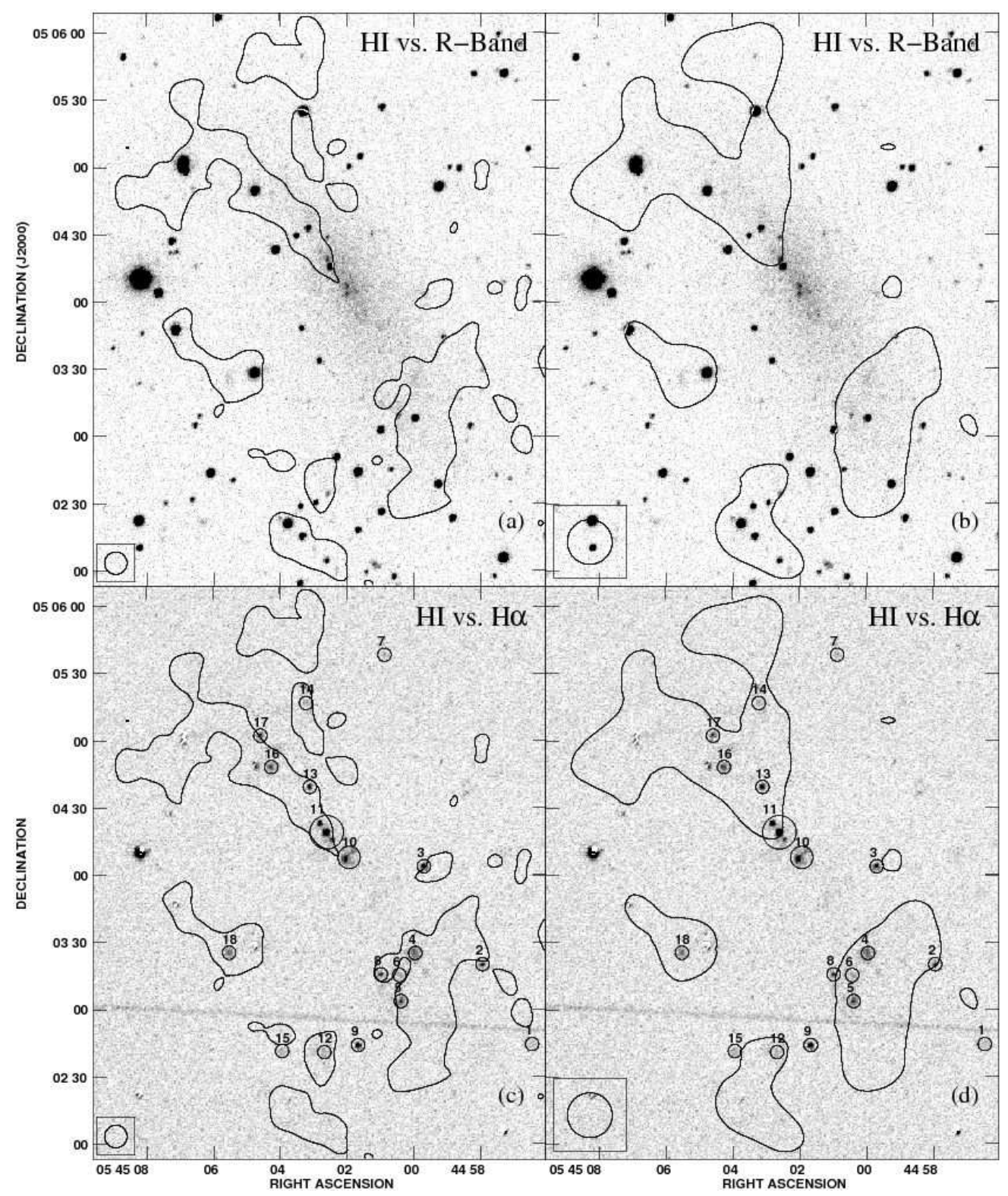

Fig. 5.- Optical images of the Orion dwarf, with the $10^{-21} \mathrm{~cm}^{-2} \mathrm{H}$ I column density contour superposed (beam size $=10^{\prime \prime}$ in panels $a$ and $c$; beam size $=20^{\prime \prime}$ in panels $b$ and $d$ ). Panels (a) and (b) show the stellar distribution; panels (c) and (d) show regions of recent $\mathrm{SF}$ as traced by continuum subtracted $\mathrm{H} \alpha$ emission. We detect 20 individual $\mathrm{H}$ II regions (circled and numbered in panels $c$ and $d$ in order of increasing Right Ascension). Note that $\mathrm{H} \mathrm{II}$ region \#11 consists of three distinct peaks that are just barely resolved; the flux in Table 2 is the sum of these three. The regions of high $\mathrm{H}$ I column density in general trace the locations of active SF; this is in agreement with the "canonical" column density threshold of $10^{21} \mathrm{~cm}^{-2}$ for active SF (e.g., Skillman 1987, Kennicutt 1989, Kennicutt 1998b, and references therein). 


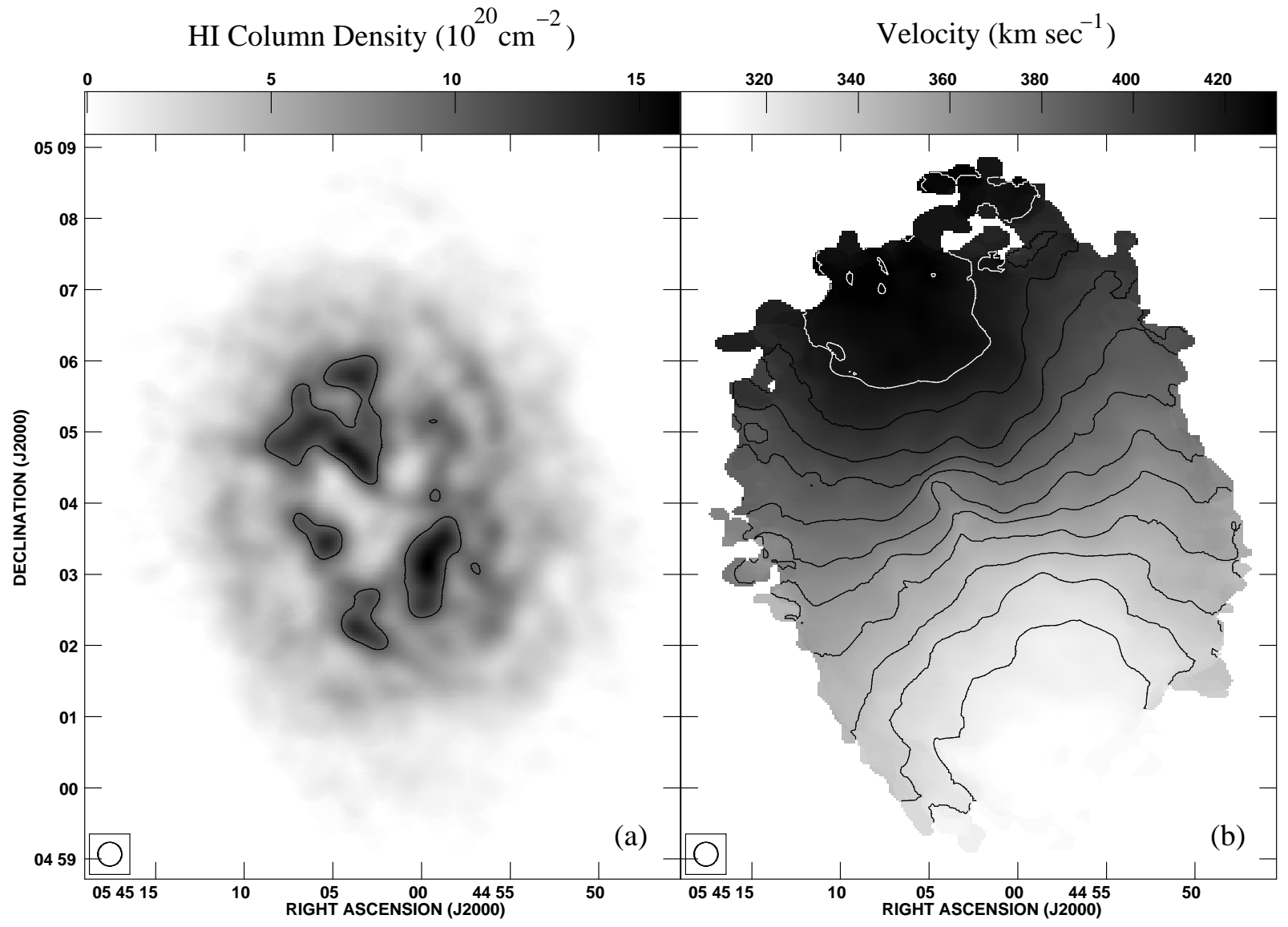

Fig. 6. - Comparison of the H I column density distribution ( $a$ ) and the integrated velocity field (b); the beam size is $20^{\prime \prime}$. The contour in $a$ is at the $10^{21} \mathrm{~cm}^{-2}$ level (c.f., Figures 4, 5). The contours in (b) show velocities between 320 and $430 \mathrm{~km} \mathrm{~s}^{-1}$, separated by $10 \mathrm{~km} \mathrm{~s}^{-1}$. The Orion dwarf is undergoing well-ordered rotation throughout most of the disk. The inner regions have prominent "hole/depression" structures; a pronounced kink in the velocity field is apparent at the same location. 


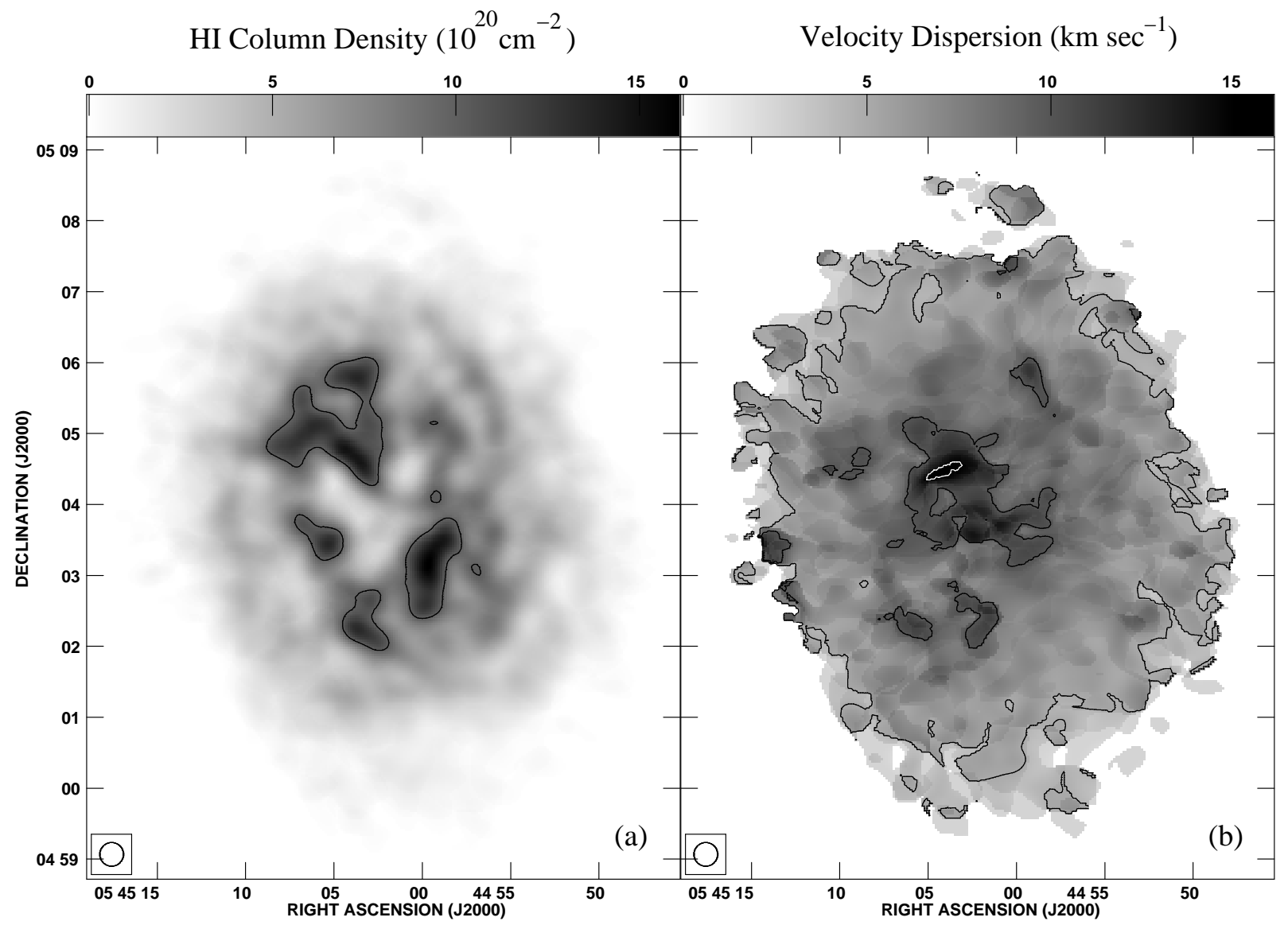

Fig. 7.- Comparison of the H I column density distribution (a) and the intensity-weighted velocity dispersion $(b)$; the beam size is $20^{\prime \prime}$. The contour in $(a)$ is at the $10^{21} \mathrm{~cm}^{-2}$ level (c.f., Figures 4, 5, 6); the contours in (b) are at the 5, 10, $15 \mathrm{~km} \mathrm{~s}^{-1}$ levels. Note the prominent increase in velocity dispersion in the inner disk. 


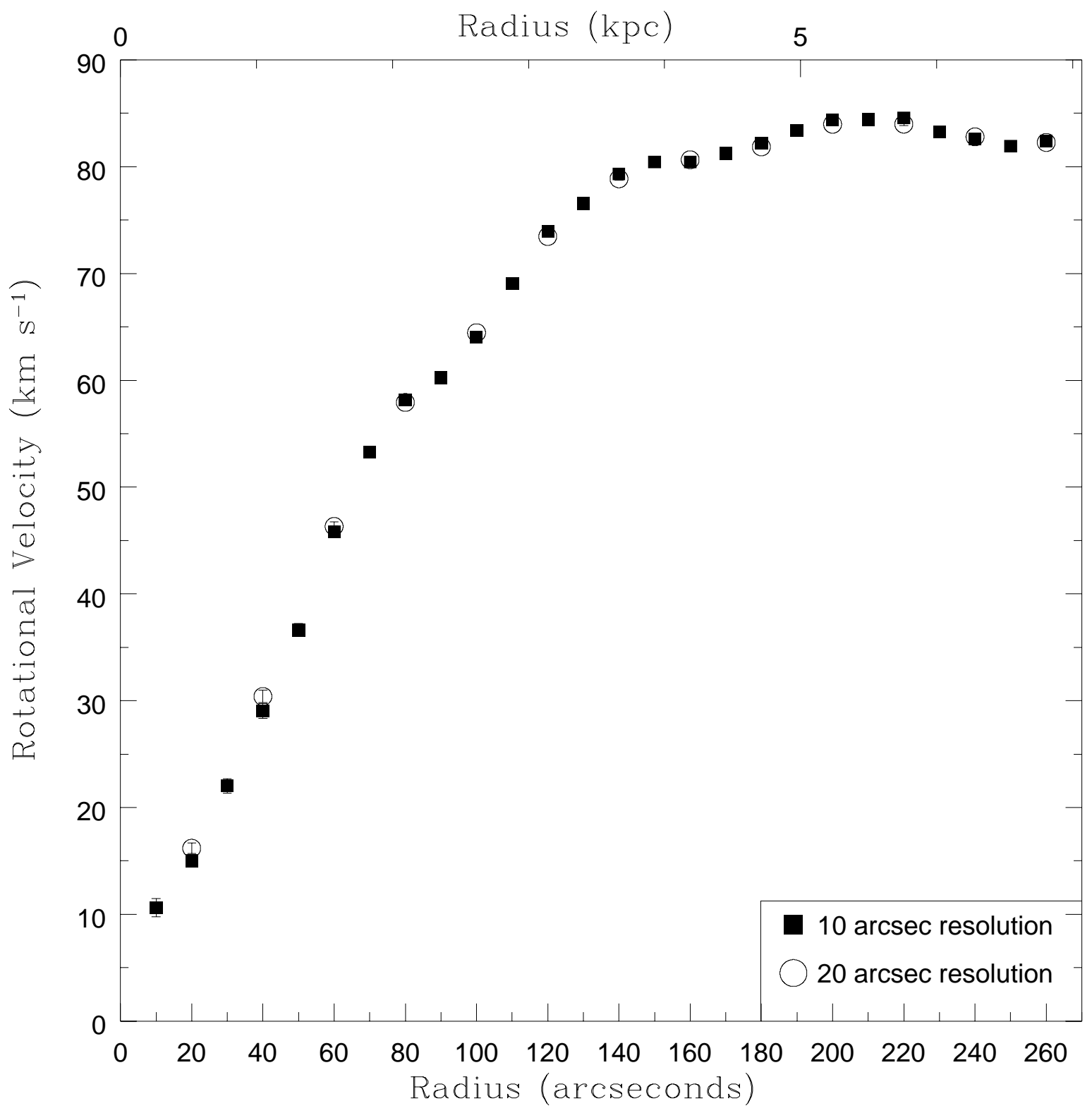

Fig. 8.- Rotation curve of the Orion dwarf galaxy, derived from both the $10^{\prime \prime}$ and $20^{\prime \prime}$ datacubes; errorbars are plotted and in most cases are smaller than the symbol sizes. At a radius of $\sim 140^{\prime \prime}(\sim 3.7 \mathrm{kpc})$ the curve flattens to $\sim 82 \mathrm{~km} \mathrm{~s}^{-1}$ and remains flat to our detection limit. The inferred dynamical mass $\left(\mathrm{M}_{\mathrm{dyn}}=10.6 \times 10^{9} \mathrm{M}_{\odot}\right)$ exceeds the mass of the gaseous and stellar components. 


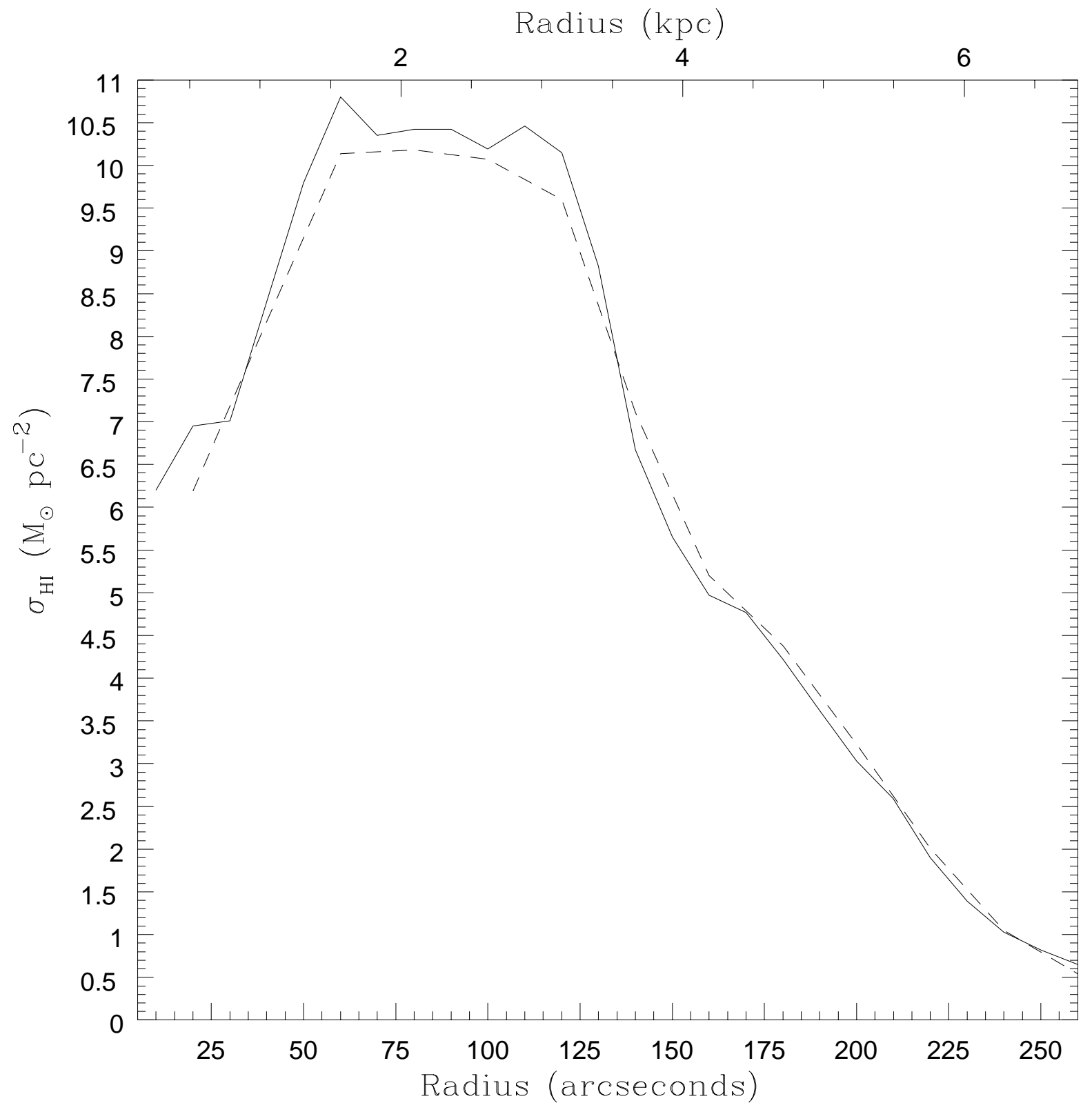

Fig. 9.- Radially averaged H I mass surface density profiles of the Orion dwarf, created by summing $\mathrm{H}$ I emission in concentric rings emanating from the dynamical center found in our rotation curve analysis. The solid line was created from the $10^{\prime \prime}$ resolution image, while the dotted line was created from the $20^{\prime \prime}$ resolution image. These profiles shows that the inner "holes/depressions" have significantly less H I gas mass per unit area than regions at intermediate radii further out in the disk. 


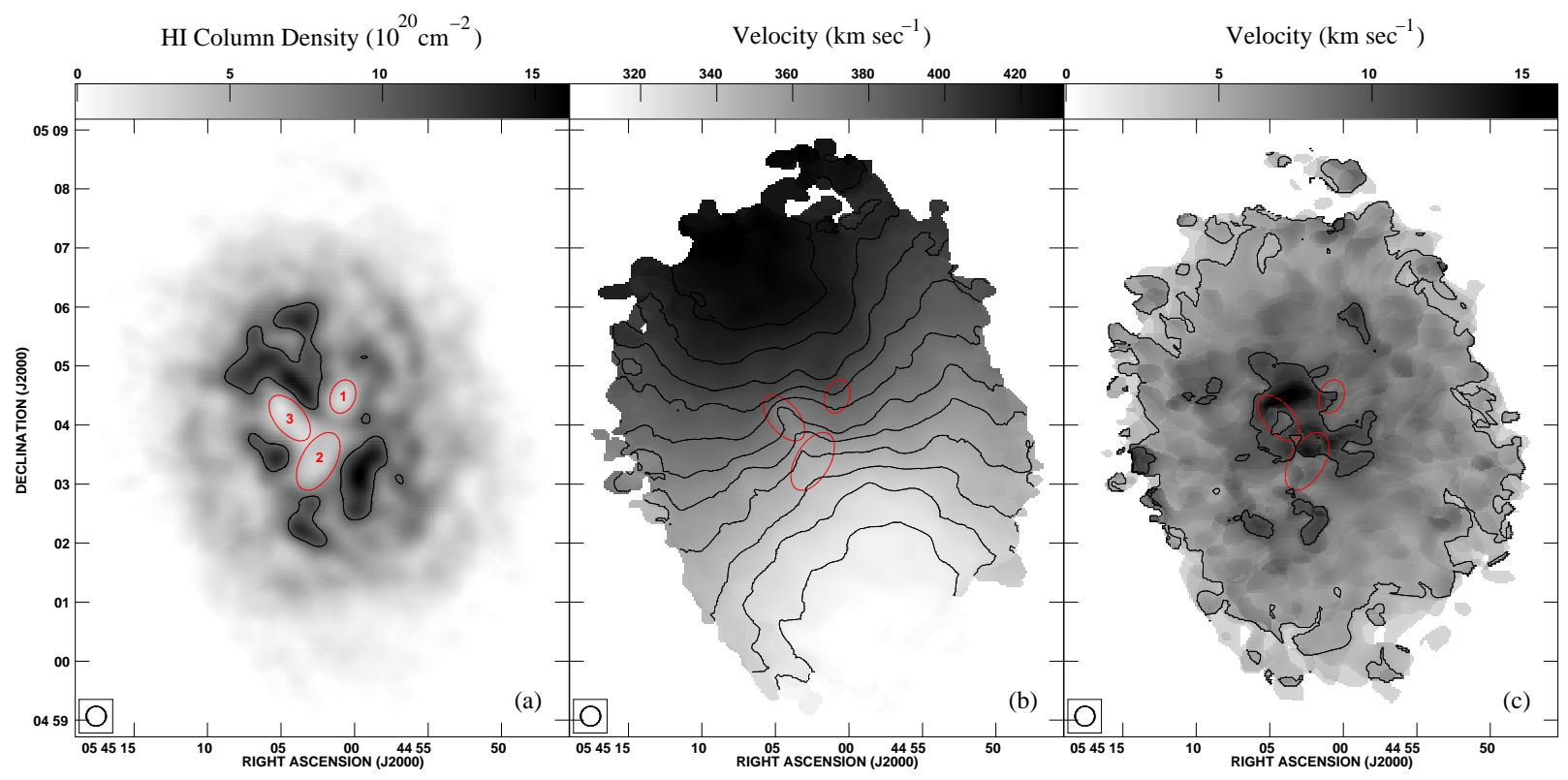

Fig. 10. - The H I column density (a), velocity field (b), and velocity dispersion $(c)$ of the Orion dwarf at $20^{\prime \prime}$ resolution. The contours in each panel are at the same levels as those shown in Figures 6 and 7 . The red ellipses shown denote the locations of the three most prominent holes/depressions in the ISM. Note that these features are concentrated in the region of elevated velocity dispersion compared to the more quiescent outer disk. Further, the departure from ordered rotation in the inner disk, prominent in $(b)$, corresponds well with the location of the easternmost of these features. 


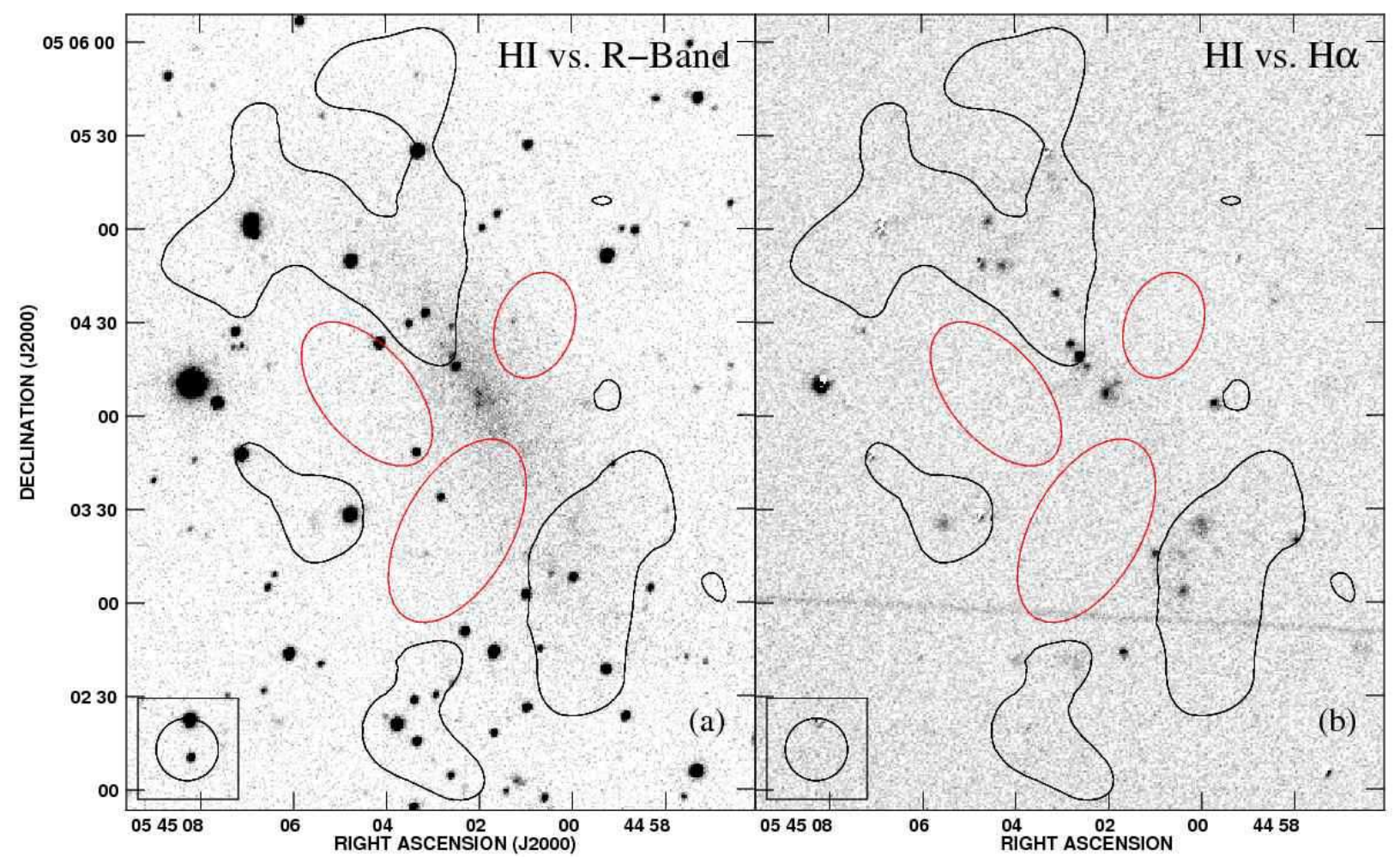

Fig. 11. - Locations of the three most prominent H I "holes/depressions" in the inner disk (shown as red ellipses; c.f. Figures [5] and 10) compared to the stellar $(a)$ and continuum subtracted $\mathrm{H} \alpha$ (b) distributions. The $10^{21} \mathrm{~cm}^{-2} \mathrm{H}$ I column density contour (20" resolution) is overlaid for reference in black. Note that the central stellar component occupies the region interior to these three features. 


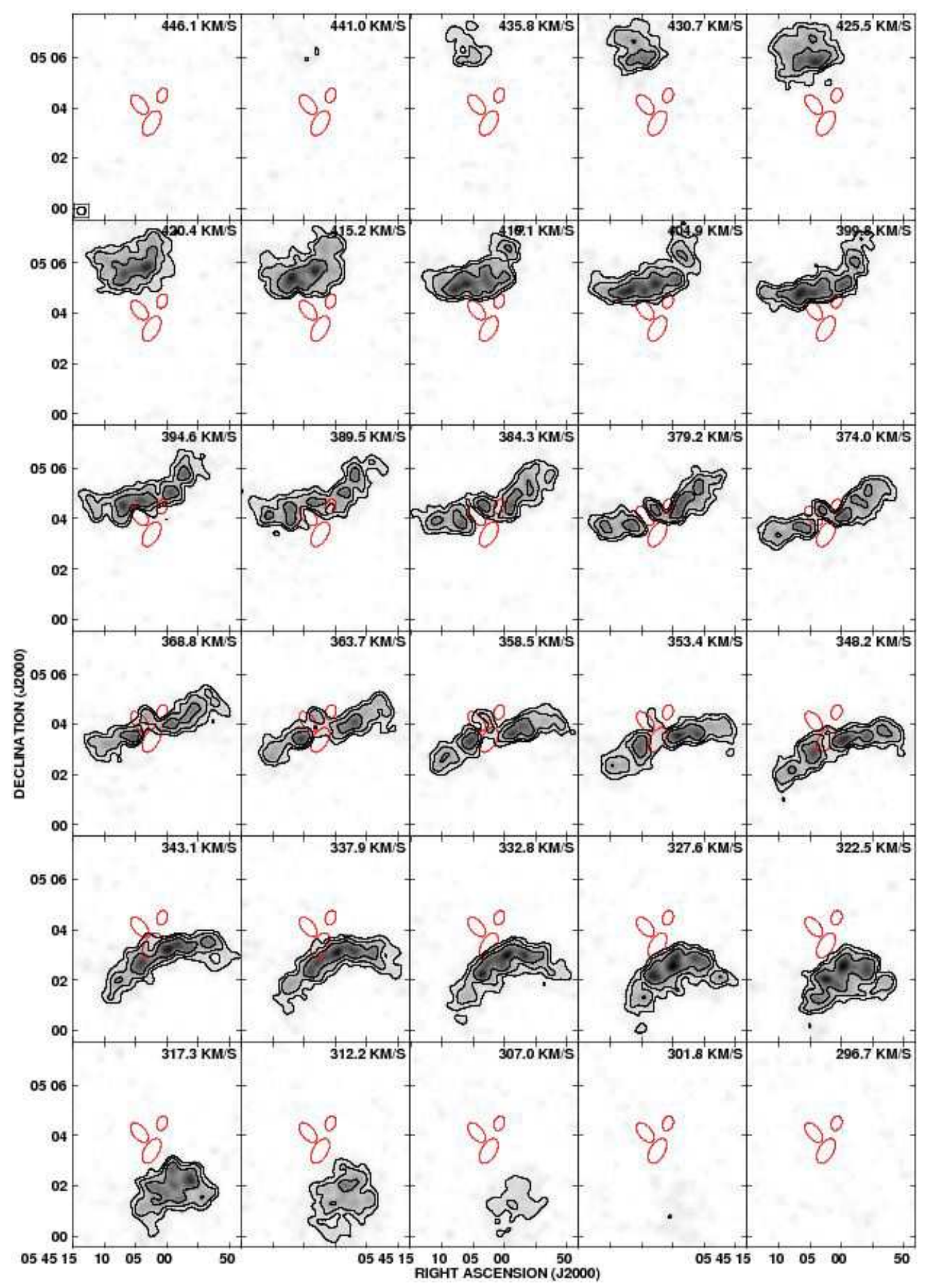

Fig. 12.- Same as Figure 2, except here the locations of the three most prominent H I "holes/depressions" are overlaid as red ellipses (c.f., Figures 10 and 11). Note that while we do not detect the signatures of expansion in these features at the current time, they are cleanly delineated in velocity space (e.g., consider the easternmost "hole/depression" feature and the void apparent in the panels centered around $\sim 380 \mathrm{~km} \mathrm{~s}^{-1}$.) 


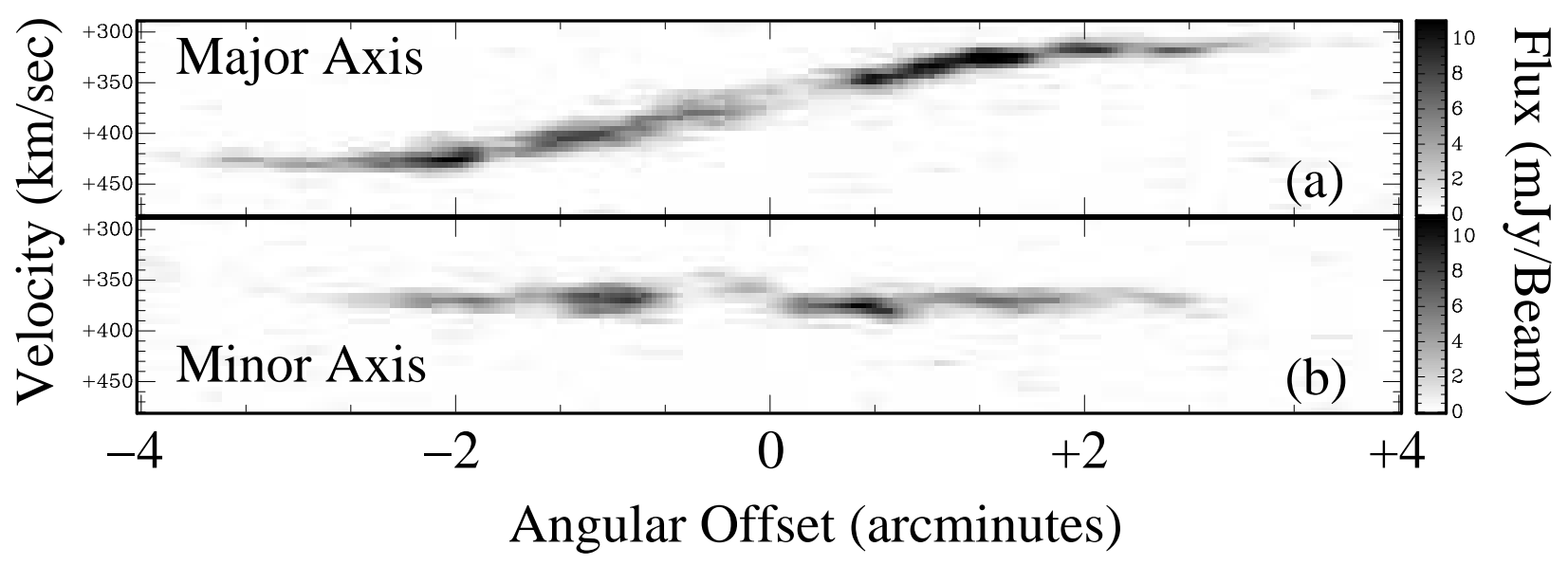

Fig. 13.- Major $\left(20^{\circ}\right.$, measured east of north; $\left.a\right)$ and minor $\left(110^{\circ}\right.$, measured east of north; b) axis position-velocity diagrams. Note the kinematic discontinuities arising from the holes/depressions in the ISM [between 0 and $+0.5^{\prime}$ in $(a)$ and between 0 and $-0.5^{\prime}$ in (b)]; these can be compared with Figures 10 and 12. Each cut is centered on the dynamical center (marked in Figure 2) of the galaxy as derived from the rotation curve analysis (see Figure 8). 